\title{
A GNSS Quadrupole Antenna With a Spatial Polarizer for the Suppression of Low-Angle Multipath
}

\author{
E. R. Gafarov, A. V. Stankovsky, Y. P. Salomatov \\ Institute of Engineering Physics and Radio Electronics \\ Siberian Federal University \\ Krasnoyarsk, Russia \\ slazen@mail.ru
}

\begin{abstract}
The article presents a quadrupole antenna with spatial meander-line polarizer to minimize multipath signal reception. The single quadrupole antenna with low axial ratio at grazing angles was considered. Modeling of the spatial meanderline polarizer for the antenna has been presented. Based on characteristics of models the conclusions of applicability in the area of global navigation satellite systems have been made.
\end{abstract}

Keywords-quadrupole antenna; meander-line polarizer; global navigation satellite system; grazing angles; right-hand circular polarization.

\section{INTRODUCTION}

At present day we know a lot of navigation satellite systems (GLONASS, GPS, GALILEO etc.). Each of these navigation systems is characterized by a closely situated operating frequency bands, operation of the systems is performed typically in a few sub bands (L1, L2, L3 (L5), etc.). Regardless of the satellite navigation system and a frequency band the undesirable effect of multipath interference is observed. Together with the direct signal from the satellite the signal reflected from the ground and other objects located near the antenna, such as buildings, a variety of props and pillars goes to the antenna input $[1,2]$. Thus, the so-called multipath reception both below and above the horizon can occur. The decrease in positioning accuracy is a consequence of the reflected signal reception.

The polarization of multipath signal is inverted from right hand to left hand as a result of reflections from the ground and other objects. In order to reduce the influence of reflected signal the receiving antenna must have a right-hand circular polarization in the reference direction and at grazing angles (angles close to the horizon). In other words the axial ratio (AR) of receiving antenna with right circular polarization must be equal to 1 over the whole upper hemisphere, as well as at the angles close to $90^{\circ}$ elevation and below, slightly overlapping the lower hemisphere [3]. If this condition is met, the signal of multipath interference by the reflection from underlying surfaces and objects in the vicinity of the antenna will have a very low level at the antenna output. Thus, the antenna acts as a polarizing spatial filter for multipath signals. It should be noted that by means of received signal post processing the multipath effect cannot be eliminated. Consequently, the suppression of multipath must be implemented directly in the antenna with the help of polarization isolation.

Classical approaches for multipath mitigation involves using of an antenna with a choke ring structure or a highimpedance surface [4, 5]. However, choke ring structure suppresses the propagation of surface waves in both the left and right-hand circular polarization at a receiving antenna. Hence, the antenna with such structure cannot have a high level of AR at the elevation angles $90^{\circ}$ and above.

Another way to eliminating multipath is using an antenna without bulky choke ring structure $[6,7]$, in which the AR at low angles can be increased by using additional techniques.

In order to achieve a high level of AR at grazing angles, it is necessary to use a polarizer [8]. Polarizer structure may be flat (for application on a patch antenna) or spatial (for use in stub antenna). In the polarizer arrangement angle sector the phase and amplitude radiation pattern of antenna must be uniform without sudden jumps and dips.

The antenna consisting of four monopoles located above the round ground plane in a single version and using a polarizer will be discussed below.

\section{ANTENNA AND POLARIZER CONFIGURATIONS}

To obtain the circularly polarized antenna with high AR and an uniform phase and amplitude radiation pattern four monopoles (a quadrupole) are supposed to be used. Monopoles should be excited with equal amplitude and a phase difference of $0^{\circ}, 90^{\circ}, 180^{\circ}, 270^{\circ}$. The quadrupoles of the antenna are located above the ground plane and can be fed directly with the help of microstrip line with a characteristic impedance of $50 \mathrm{Ohms}$. In this article the design of power divider with operating range $1.15-1.65 \mathrm{GHz}$ and a phase difference in four subsequent outputs of $90^{\circ}$ was not provided. However, such a divider can be designed by means of the principles and approaches which are well known at present day. 
The antenna based on the four monopoles provides operation in all ranges of satellite navigation systems with high level of AR in the upper hemisphere. However, single antenna does not ensure high level of AR at grazing angles.

In order to increase AR at elevation angles of $80^{\circ}-110^{\circ}$ the most promising approach is using of a meander-line polarizer of cylindrical shape.

Meander-line polarizer is a set of thin metallic structures in the form of a meander-line (Fig. 1). Operation principle of the polarizer is described in [8].

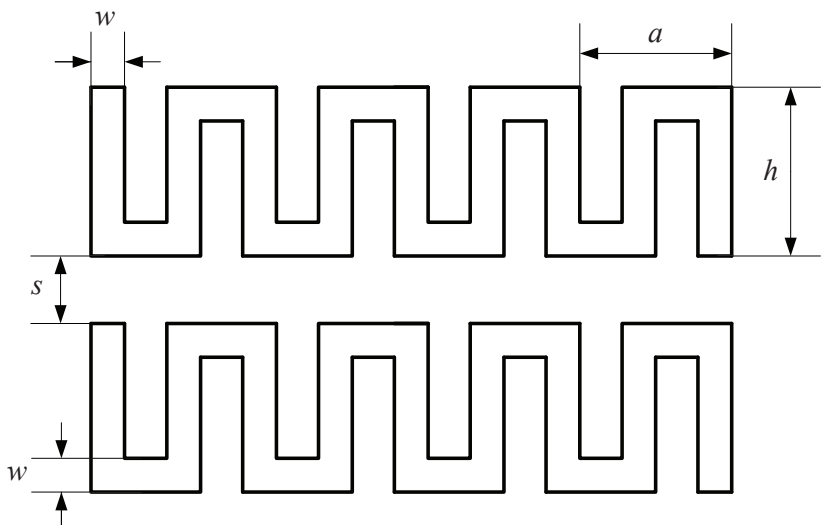

Fig. 1. Dimensions of meander-line polarizer.

In cylindrical polarizer the parameters are directly depend on the length of the cylinder circumference, i.e. on its diameter $(d)$. The distance between the strips of meanders $(s)$ is calculated as follows:

$$
s=\sqrt{\left(\frac{d \cdot \pi}{N}\right)^{2} \cdot \frac{1}{2}}-h .
$$

The number of meander strips equals 10 . They are located at every $36^{\circ}$. Dimensions of polarizer are following: $w=4.5 \mathrm{~mm}, a=21.5 \mathrm{~mm}, h=31 \mathrm{~mm}, k=120 \mathrm{~mm}$ and $d=240 \mathrm{~mm}$. To choose from the right hand and left hand circular polarization the angle of slope has to be either $+45^{\circ}$ or $-45^{\circ}$ from the reference direction.

Geometry of proposed antenna with a polarizer is represented in Fig. 2, Fig. 3.

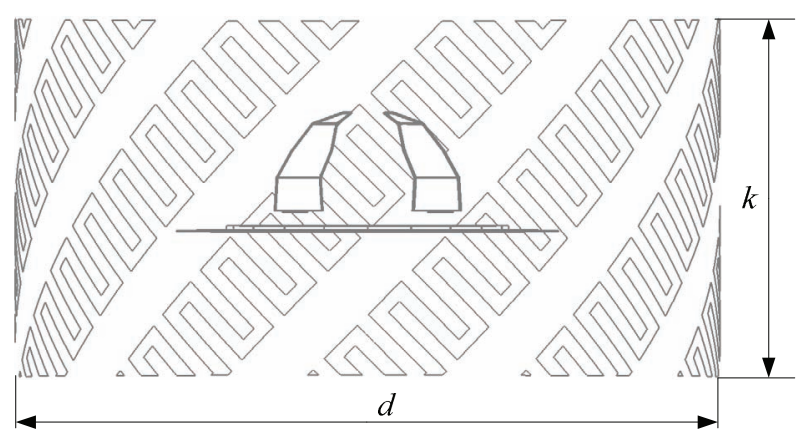

Fig. 2. Quadrupole antenna and polarizer, side view.
Cylindrical polarizer and quadrupole antenna share a common geometric center. The value of polarizer height $k$ is based on high level of AR at grazing angles. Polarizer covers the range of elevation angles $90^{\circ} \pm 20^{\circ}$ to antenna.

The parameters of polarizer strips were obtained using optimization model with quadrupole antenna on the criterion of the largest AR at grazing angles.

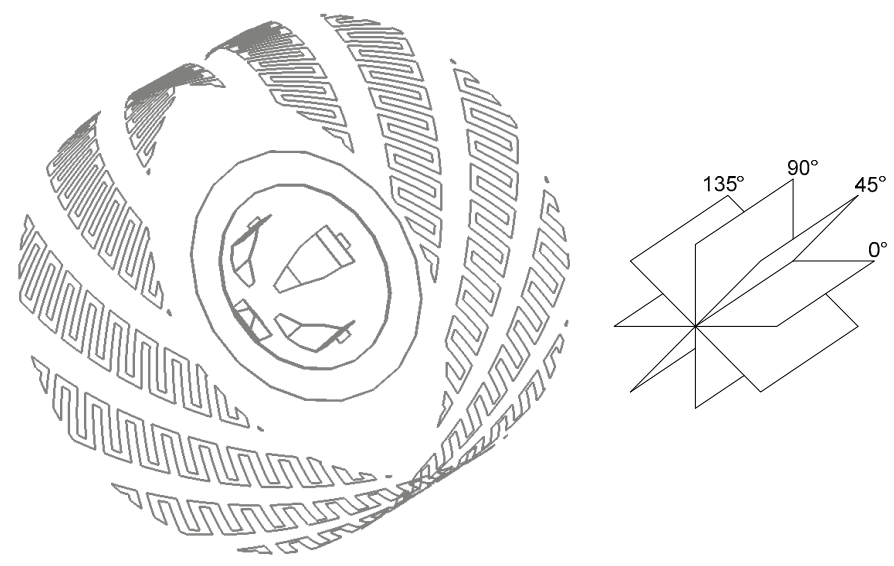

Fig. 3. 3D view of antenna and investigated planes of azimut angles (phi).

Further the simulation results of $\mathrm{AR}$ at azimuth angles within the range $\pm 120^{\circ}$ and antenna radiation patterns at two frequencies will be presented.

\section{FIELD ANALISYS}

The single-layer circular polarizer presented here is designed to operate in the frequency range L1. To expand the polarizer bandwidth or to obtain multi-frequency capability it is possible to add layers following each other with a certain radius [9]. However, due to the homogeneous and permanent polarizer step it is necessary that phase and amplitude radiation patterns would be uniform to achieve a high level of AR. Radiation patterns should be free of abrupt jumps and dips within the range of angles where the polarizer will be installed. Consequently, the AR must have similar values too within the range of angles where the polarizer is set. This property is very important for the construction of polarizer.

So as shown in Fig. 5, for a single antenna the AR at frequency of $1.6 \mathrm{GHz}$ and elevation angles in the range of $90^{\circ}$ $\pm 20^{\circ}$ is equal $0.4-0.5$. Presented curve is smooth enough and have not jumps. This AR provides isolation of the right and left-hand circular polarization of antenna about 8-9 dB. Fig. 4 shows that $\mathrm{AR}$ at frequency $1.2 \mathrm{GHz}$ is high for elevation angles $110^{\circ}$ and below. Application of polarizer in the range L2 is inconclusive due to the fact that the AR curve falls down to 0 at angles above $95^{\circ}$. Therefore, only a single layer of the cylindrical polarizer designed for the frequency of $1.6 \mathrm{GHz}$ was used.

At the frequency $1.2 \mathrm{GHz}$, the polarizer has little effect on the AR performance. Nevertheless, the AR is high in the corners close to $90^{\circ}$ (Fig. 6).

After adding to the polarizer the quadrupole antenna AR at frequency $1.6 \mathrm{GHz}$ within elevation angles $90^{\circ} \pm 20^{\circ}$ is 0.78 - 
0.95 , that is characterized by polarization decoupling 19-30 $\mathrm{dB}$ (Fig. 7). Such advantage raises the signal over the interference by $19 \mathrm{~dB}$ and more and will provide a good suppression of multipath. In addition, a high level of signal amplification with the right circular polarization at low elevation angles $\left(-3 \ldots-5 \mathrm{~dB}\right.$. from max. by $90^{\circ}$, Fig. 8$)$ allows to perform certain signal reception from satellites located in low orbits. This cannot be said about the antenna with the classic choke ring multipath signals reducing structure, in that case the right-hand circular and left circular polarization are at a close level about $-15 \ldots-20 \mathrm{~dB}$.

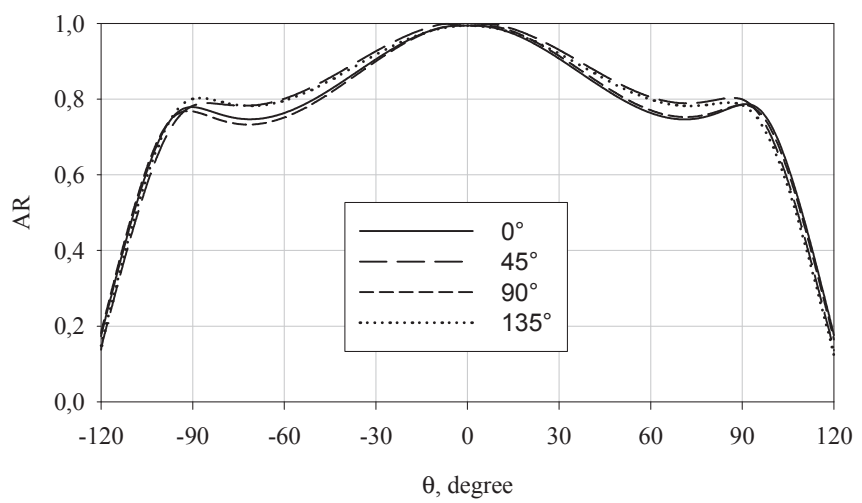

Fig. 4. AR of single antenna at $1.2 \mathrm{GHz}$.

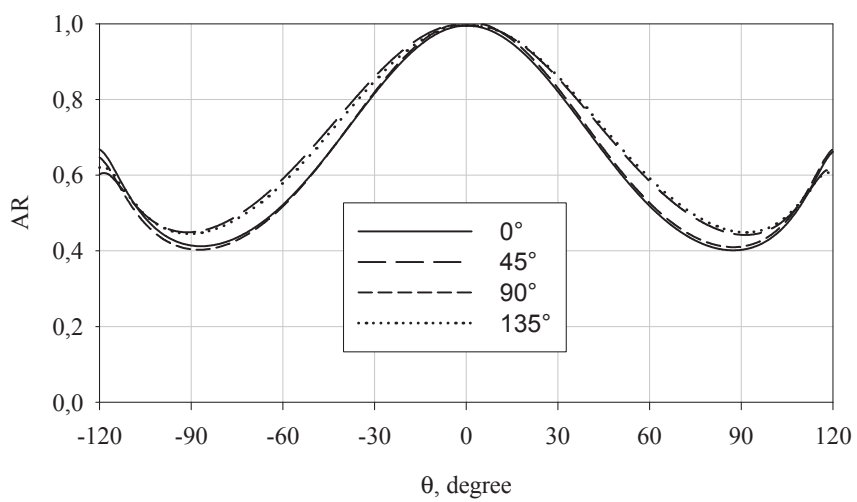

Fig. 5. AR of single antenna at $1.6 \mathrm{GHz}$.

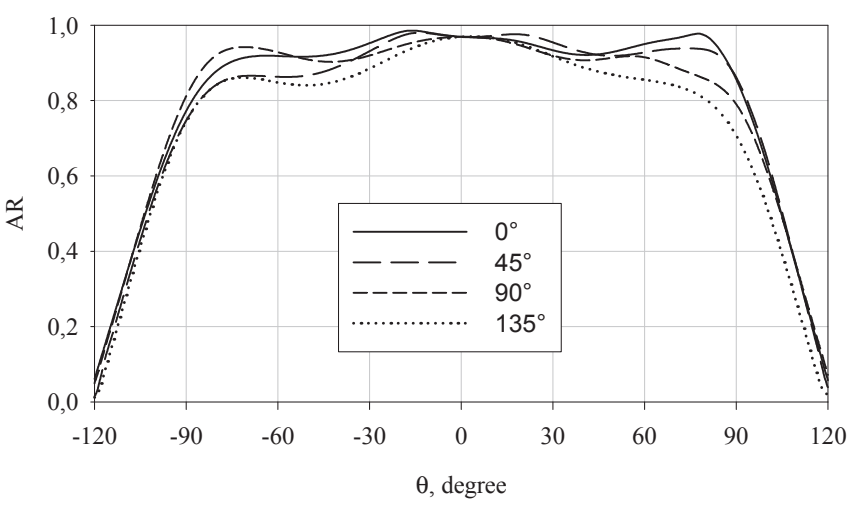

Fig. 6. AR of antenna with polarizer at $1.2 \mathrm{GHz}$.

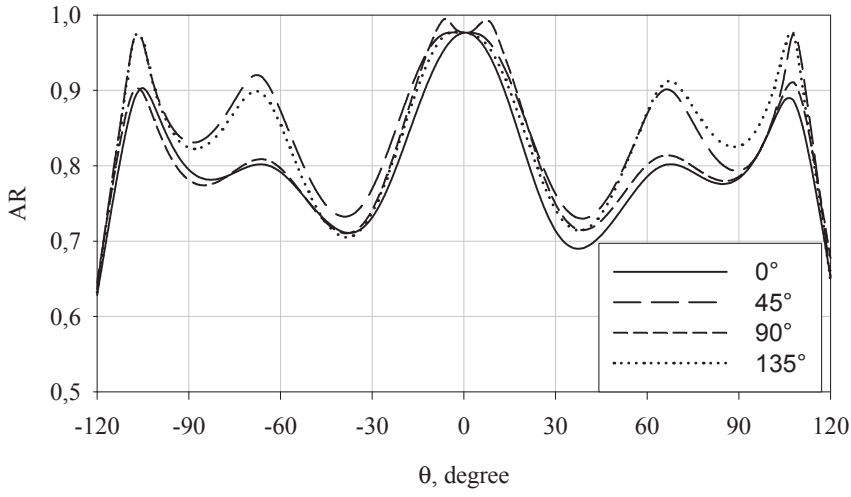

Fig. 7. AR of antenna with polarizer at $1.6 \mathrm{GHz}$.

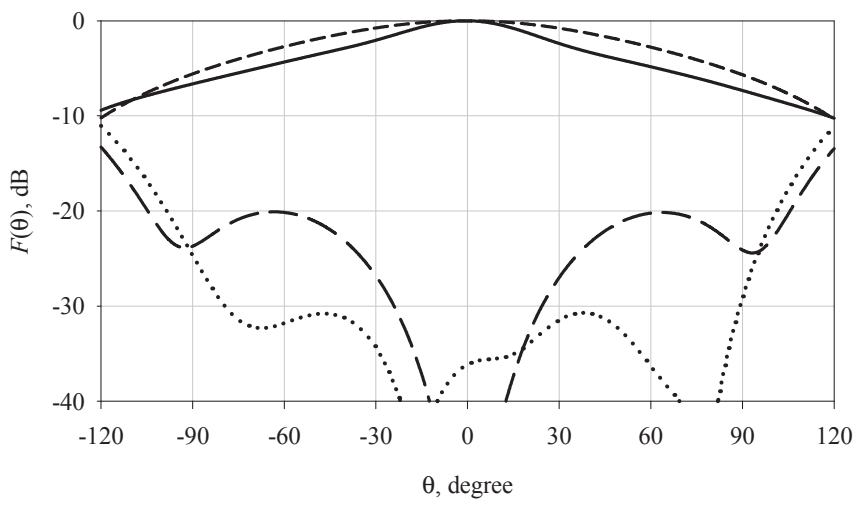

(a)

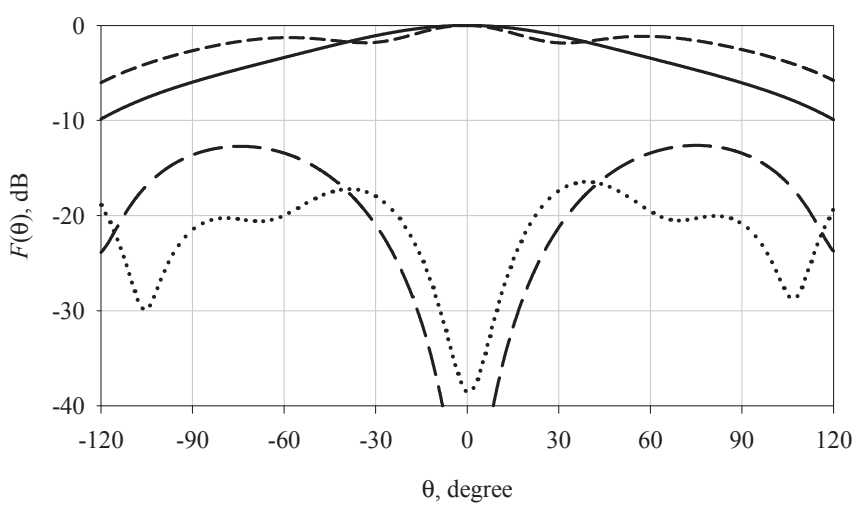

(b)

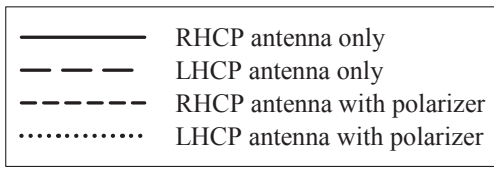

Fig. 8. Radiation pattern of RHCP and LHCP for antenna with polarizer at $1.2 \mathrm{GHz}(\mathrm{a}), 1.6 \mathrm{GHz}(\mathrm{b})$, plane: $\mathrm{phi}=0^{\circ}$.

The antenna simulation results with a polarizer calculated for the L1 frequency range are presented. To feeding the monopoles of antenna the equal amplitudes and phase differences $0^{\circ}, 90^{\circ}, 180^{\circ}, 270^{\circ}$ are strictly specified. While the difference between the amplitudes at the sources of monopoles equals $10 \%$ and a phase deviation from strict values at $10^{\circ}$ - 
$15^{\circ}$, the AR can be reduced up to 0.7 instead of 0.8 . In other words, the right to left ratio will be reduced by $5 \mathrm{~dB}$. For this type of antenna it is important to keep the uniform amplitude division with strict phases. In addition to the above, the polarizer increases AR from 0.4 up to 0.8 at frequency 1.6 $\mathrm{GHz}$ with respect to a single antenna. However, another polarizer can be constructed with a different aspect ratio of meander-line, which will increase the AR up to 0.8 or higher for a linearly polarized antenna for use in navigation system. The main thing is the observance of the conditions of small amplitudes and phase differences in the required angle sector of polarizer.

\section{CONCLUSIONS}

The cylindrical shaped meander-line polarizer based on quadrupole antenna with right circular polarization for improving of AR at grazing angles was constructed. The results show significant advantage in increasing the RHCP/LHCP ratio for antenna with a polarizer against a single antenna. It should be noted that the spatial polarizer does not change the AR of antenna outside working range of elevation angles. Conclusions about the applicability of the polarizer on the basis of the initial characteristics of a single antenna were made. Meander-line polarizer can be used in applications to other types of single antennas.

Thus, the antenna system to mitigate multipath interference at the grazing angles with high reception level for the basic type of polarization was obtained.

\section{REFERENCES}

[1] F. Scire-Scappuzzo and S. N. Makarov, "A low-multipath GPS wideband antenna with cutoff or non-cutoff corrugated ground plane", IEEE Trans. Antennas Propag., vol. 57, no. 1, pp. 33-46, Jan. 2009.

[2] L. I. Basilio, R. L. Chen, J. T. Williams, and D. R. Jackson, "A new planar dual-band GPS antenna designed for reduced susceptibility to low-angle multipath", IEEE Trans. Antennas Propag., vol. 55, no. 8, pp. 2358-2366, Aug. 2007.

[3] F. Khosravi, H. Moghadas, P. Mousavi, "A GNSS Antenna With a Polarization Selective Surface for the Mitigation of Low-Angle Multipath Interference", 2015 IEEE Transactions on antennas and propagation, 2015, Vol. 63, No. 12, pp. 5287-5295.

[4] J. Ashjaee, V. S. Filippov, D. V. Tatarnikov, A. V. Astakhov, and I. V. Sutjagin, "Dual-frequency choke-ring ground planes," US Patent 6278 407, Aug. 21, 2001.

[5] R. Baggen, M. Martínez-Vázquez, J. Leiss, S. Holzwarth, L. S. Drioli, and P. de Maagt, "Low profile GALILEO antenna using EBG technology", IEEE Trans. Antennas Propag., vol. 56, no. 3, pp. 667674, Mar. 2008.

[6] NovaTel Inc. (Oct. 2013). GPS-704X Antenna Design and Performance [Online]. Available: http://www.novatel.com/assets/Documents/Papers/ GPS-704xWhitePaper.pdf

[7] E. R. Gafarov, Y. P. Salomatov, "The dual-band antenna for navigation satellite systems", Microwave\&Telecommunication Technology (CriMiCo), 2015 25th International Crimean Conference, Sevastopol, 713 september, 2015.

[8] Leo Young, Lloyd A. Robinson, Colin A. Hacking. Meander-Line Polarizer// 1973 IEEE Transactions on Antennas and Propagation, May 1973.

[9] Ruey-Shi Chu, Kuan-Min Lee. Analytical Model of a Multilayered Meander-Line Polarizer Plate with Normal and Oblique Plane-Wave Incidence // 1987 IEEE Transactions on Antennas and Propagation, vol. AP-35, No. 6, June 1987. P. 652-661. 